\title{
Notch2 governs the rate of generation of mouse long- and short-term repopulating stem cells
}

\author{
Barbara Varnum-Finney, ${ }^{1}$ Lia M. Halasz, ${ }^{1}$ Mingyi Sun, ${ }^{1}$ Thomas Gridley, ${ }^{2}$ \\ Freddy Radtke, ${ }^{3}$ and Irwin D. Bernstein ${ }^{1}$
}

${ }^{1}$ Pediatric Oncology, Clinical Division, Fred Hutchinson Cancer Research Center, Seattle, Washington, USA. ${ }^{2}$ The Jackson Laboratory, Bar Harbor, Maine, USA ${ }^{3}$ Ludwig Institute for Cancer Research, University of Lausanne, Epalinges, Switzerland.

\begin{abstract}
HSCs either self-renew or differentiate to give rise to multipotent cells whose progeny provide blood cell precursors. However, surprisingly little is known about the factors that regulate this choice of self-renewal versus differentiation. One candidate is the Notch signaling pathway, with ex vivo studies suggesting that Notch regulates HSC differentiation, although a functional role for Notch in HSC self-renewal in vivo remains controversial. Here, we have shown that Notch2, and not Notch1, inhibits myeloid differentiation and enhances generation of primitive Sca- $1^{+} \mathrm{c}-\mathrm{kit}^{+}$progenitors following in vitro culture of enriched HSCs with purified Notch ligands. In mice, Notch2 enhanced the rate of formation of short-term repopulating multipotential progenitor cells (MPPs) as well as long-term repopulating HSCs, while delaying myeloid differentiation in BM following injury. However, consistent with previous reports, once homeostasis was achieved, neither Notch1 nor Notch2 affected repopulating cell self-renewal. These data indicate a Notch2-dependent role in assuring orderly repopulation by HSCs, MPPs, myeloid cells, and lymphoid cells during BM regeneration.
\end{abstract}

\section{Introduction}

HSCs either self-renew or give rise to multipotent cells whose progeny provide precursors committed to the lymphoid and myeloid lineages. Recent studies have identified factors that maintain HSCs in a quiescent state required for long-term maintenance or that induce their proliferation $(1,2)$. However, little is known about factors that regulate HSC choice of self-renewal versus differentiation. One candidate, the Notch pathway, is known to affect self-renewal and lineage fate of precursor cells in numerous developing systems, including in neural, muscle, and skin precursors (3). Within the hematopoietic system, Notch directs many lineage choices, including Notch1-mediated megakaryocyte specification (4). In the thymus, Notch 1 promotes T cell differentiation at the expense of B cell differentiation, while in the spleen, Notch2 mediates mast cell fate and promotes generation of B cell subsets (5-9). Nonetheless, although Notch receptors are expressed by HSCs and their progeny, and Notch ligands are expressed in surrounding niches in BM, studies involving in vivo deletion or inhibition of Notch pathway components have not convincingly demonstrated an in vivo effect of Notch signaling on HSC self-renewal and differentiation (10-12).

Ex vivo studies have shown that overexpression of constitutively active Notch 1 intracellular domain or the Notch downstream target Hes1, or incubation of HSCs with cell-expressed or purified recombinant Notch ligands, leads to inhibition of myeloid differentiation while enhancing multipotential progenitor cell (MPP) generation, resulting in increased numbers of MPPs capable of short-term repopulation (13-18). More recently, Butler et al. achieved Notch-dependent self-renewal of HSCs upon cocul-

Authorship note: Barbara Varnum-Finney and Lia M. Halasz contributed equally to this work.

Conflict of interest: The authors have declared that no conflict of interest exists. Citation for this article: J Clin Invest. 2011;121(3):1207-1216. doi:10.1172/JCI43868. ture with adenovirus-infected endothelial cell lines (18). These results suggest that failure to identify an in vivo role for Notch signaling in HSC/MPP function reflects compensatory effects of other factors, such as hematopoietic growth factors, that ensure appropriate generation of mature blood elements. They also raise the possibility that Notch-driven events are recapitulated in vivo under nonhomeostatic conditions, in which compensatory mechanisms may not mask Notch-induced effects on HSC/MPP growth and differentiation.

Our previous ex vivo studies suggest that alternative cell fates induced by Notch activation result from quantitative differences in Notch signaling (19). Ex vivo culture of BM-derived HSCs/ MPPs with different densities of purified Notch ligands revealed that both low and high levels of Notch signaling inhibit myeloid differentiation and enhance generation of MPPs, but only low levels of Notch signaling enhance generation of B220 cells capable of rapid B cell differentiation after coculture with OP-9 cells. High levels of Notch signaling inhibit generation of these B220 ${ }^{+}$ $\mathrm{B}$ cell precursors and instead enhance generation of CD25+Thy $1^{+}$ $\mathrm{T}$ cell precursors.

Although in vivo mechanisms underlying quantitative regulation of Notch signaling are not fully understood, ex vivo studies suggest that individual Notch receptors have different transcriptional activating ability (20); thus, selective paralog usage can determine the extent of Notch target gene activation. This selective activation of specific Notch paralogs can result from interaction with specific ligands, such as selective activation of Notch2 by Jagged family members in the presence of Fringe, a glycosyltransferase that mediates differential receptor activation by unique ligands in both Drosophila and mammals (21-25). Overall, these studies have raised questions of distinct function of individual Notch paralogs in HSC function.

Here, we identified distinct roles for Notch1 and Notch2 receptors in modulating HSC/MPP growth and differentiation, both ex vivo during culture with purified ligands and in vivo during 
stress hematopoiesis. Using mice bearing deletions of either Notch1 or Notch2, our ex vivo studies revealed that either Jagged1 or Delta1 selectively activated Notch 2 in the HSC-enriched quiescent side population of $\mathrm{Lin}^{-} \mathrm{Sca}-1^{+} \mathrm{c}-\mathrm{kit}^{+}$(LSK) cells (referred to herein as LSKSP cells) to inhibit myeloid differentiation and enhance generation of MPP. Our in vivo findings supported previous publications showing no effect of Notch signaling on long-term repopulating HSC self-renewal when assessed during homeostasis (12), but also revealed Notch2-dependent inhibition of myeloid differentiation and an enhanced rate of generation of both short- (MPP) and long-term (HSC) repopulating cells during stress hematopoiesis. These results demonstrated a role for Notch signaling in impeding myeloid differentiation and enhancing HSC/MPP formation, assuring orderly development during nonhomeostatic hematopoiesis.

\section{Results}

Notch ligand inbibition of myeloid differentiation and enhanced generation of $\mathrm{SK}^{+}$cells occurs in the quiescent LSKSP BM fraction. We previously showed that immobilized Notch ligand Delta $1^{\text {ext-IgG }}$ inhibits default myeloid differentiation by $\mathrm{Lin}^{-} \mathrm{Sca}-1^{+} \mathrm{c}-\mathrm{kit}^{+}-$ enriched (LSK-enriched) BM cells and instead induces a multilog-increased number of progeny coexpressing Sca-1 and c-kit $\left(\mathrm{SK}^{+}\right.$; ref. 26). To determine whether Notch signaling acts directly on an HSC-enriched population, we further enriched for HSCs based on a Hoechst 33342 dye side population efflux profile. We then cultured the isolated the side population and non-side population fractions of LSK cells with engineered Notch ligands consisting of the extracellular domains of Delta 1 or Jagged 1 fused to the Fc domain of human IgG1 (Delta $1^{\text {ext-IgG }}$ and Jagged $1^{\text {ext-IgG }}$ ) immobilized with fibronectin fragments on the plastic surfaces of tissue culture wells and in the presence of soluble cytokines stem cell factor (SCF), IL-6, Flt3-L, and IL-11. As shown in Figure 1, A and B, more than $80 \%$ of the progeny generated by LSKSP cells after 3 and 7 days of culture with either ligand were $\mathrm{SK}^{+}$and did not coexpress myeloid antigens GR1 and F4/80. Furthermore, more than $80 \%$ of the progeny were still $\mathrm{SK}^{+}$after 14 days, when the 1,000 original LSKSP cells generated greater than $10^{10} \mathrm{SK}^{+}$progeny (data not shown). In contrast, the non-side population fraction of LSK progenitors failed to undergo $\mathrm{SK}^{+}$generation with either ligand, and few progeny of the initial 1,000 non-side population LSK cells remained viable after 7 days (data not shown). With control substrate human IgG, LSKSP and non-side population LSK cells generated similar numbers and proportions of myeloid progeny, but generated reduced proportions of $\mathrm{SK}^{+}$progeny compared with cells incubated with Notch ligand (Figure 1, A and B, and data not shown). Furthermore, $\mathrm{SK}^{+}$progeny of LSKSP cells after 7 days of culture with human IgG coexpressed myeloid antigen F4/80 (Figure 1B). These results indicated that the BM LSK cells responding to Notch ligands with enhanced generation of $\mathrm{SK}^{+}$ cells are contained in the HSC-enriched $\mathrm{SP}^{+}$fraction.

To test induction of Notch signaling by ligand, we measured expression of the Notch target Hes 1 and found 3-fold increased expression after a 3-hour incubation of LSKSP cells with either Delta $1^{\text {ext-IgG }}$ or Jagged $1^{\text {ext-IgG }}$ (Figure 1C, control). Moreover, analysis of mRNA expression of Cebpa, a key regulator of myeloid differentiation, showed that LSKSP cells cultured for 3 hours with human IgG expressed 3-fold more Cebpa mRNA copies than did LSKSP cells cultured for 3 hours with Delta $1^{\text {ext-IgG }}$ or
Jagged $1^{\text {ext-IgG }}$ (Figure 1D, control). Thus, even at this very early time point, Notch signaling inhibited induction of a myeloid differentiation program.

Selective activation of Notch2 mediates inbibition of myeloid differentiation and enhanced generation of $\mathrm{SK}^{+}$cells. To identify specific Notch receptors involved in myeloid inhibition and $\mathrm{SK}^{+}$generation, we monitored differentiation of Notch-deficient LSKSP cells during culture with Notch ligands. Because both Notch1 and Notch2 deletion results in embryonic lethality, we induced receptor deletions using mice bearing floxed Notch1 or Notch2 genes and an MxCre gene (Notch $1^{f l / f l} \mathrm{Cr} e^{+}$or $\left.N o t c h 2^{f l / f l} \mathrm{Cr} e^{+}\right)$. Notch1 or Notch2 deletions were induced in vivo in young mice with multiple injections of the interferon inducer poly-I/C. At 1-2 weeks after the last polyI/C injection, Notch1 or Notch2 copy number in genomic DNA from peripheral blood (PB) cells from deficient animals $\left(\mathrm{Cre}^{+}\right)$was less than $10 \%$ of that in PB cells from control animals $\left(\mathrm{Cre}^{-}\right)$, as assessed by quantitative genomic PCR. For culture experiments, Notch deletions were also induced ex vivo by infecting sorted LSKSP cells from Notch $1^{f l / f l}$ or Notch $2^{f l / f l}$ mice with lentivirus encoding Cre recombinase. Similar results were obtained with either method of gene deletion. Notch1-deficient LSKSP cells generated a multi-log increase in number of $\mathrm{SK}^{+}$progeny in response to Notch ligands, similar to control LSKSP cells, whereas few Notch1-deficient LSKSP progeny expressed GR1 or F4/80 (Figure 1E and data not shown), which indicates that Notch1 does not mediate ligand-induced $\mathrm{SK}^{+}$ generation. In striking contrast, Notch2-deficient LSKSP cells differentiated and did not undergo $\mathrm{SK}^{+}$generation, with only $23 \%$ of the Notch2-deficient progeny being $\mathrm{SK}^{+} 7$ days after incubation with Notch ligands, similar to wild-type LSKSP cells cultured with human IgG (Figure 1E and data not shown). Furthermore, after 7 days, many Notch2-deficient $\mathrm{SK}^{+}$cells incubated with Notch ligands also expressed F4/80 (Figure 1E), and after 14 days, most Notch2-deficient progeny with Notch ligands were no longer $\mathrm{SK}^{+}$, but were $\mathrm{GR} 1^{+}$and/or F4/80+ (Figure $1 \mathrm{~F}$ ).

To determine the basis for the selective function of Notch 2 in LSKSP cells, we assessed Hes 1 expression during culture of LSKSP cells with Notch ligand. After 3 hours of culture, increased Hes 1 mRNA expression was observed in Notch1-deficient and control cells, but not in Notch2-deficient cells (Figure 1C). Similarly, reduced Cebpa expression was seen in Notch1-deficient cells, but not Notch2-deficient cells (Figure 1D and data not shown). As expected, after 7 days of culture, Notch2-deficient cells expressed increased levels of Cebpa mRNA (data not shown). Within the $\mathrm{SK}^{+}$ precursors, we found 4-fold higher Cebpa mRNA levels in Notch2deficient cells than in control cells (Figure 1G), indicative of rapid precursor differentiation along the myeloid lineage. Together, these findings indicate that Notch2 signaling inhibits myeloid differentiation by LSKSP progeny, perhaps by Hes1-mediated suppression of Cebpa transcriptional activity.

Assessment of whether selective signaling via Notch2 is dependent on insufficient expression of Notch1 in LSKSP cells revealed a 40 -fold decrease in Notch1 mRNA expression compared with Notch2 in freshly isolated LSKSP cells (data not shown). Although these data suggest that lack of Notch1-mediated signaling in LSKSP cells may result from its decreased expression, LSKSP cells cultured with Notch ligand revealed rapid Notch2dependent upregulation of Notch1 mRNA (Figure 1, H and I), which suggests that one or more alternative mechanisms, such as selective ligand use, may be required to maintain selective Notch2 activation in developing HSC progeny. 

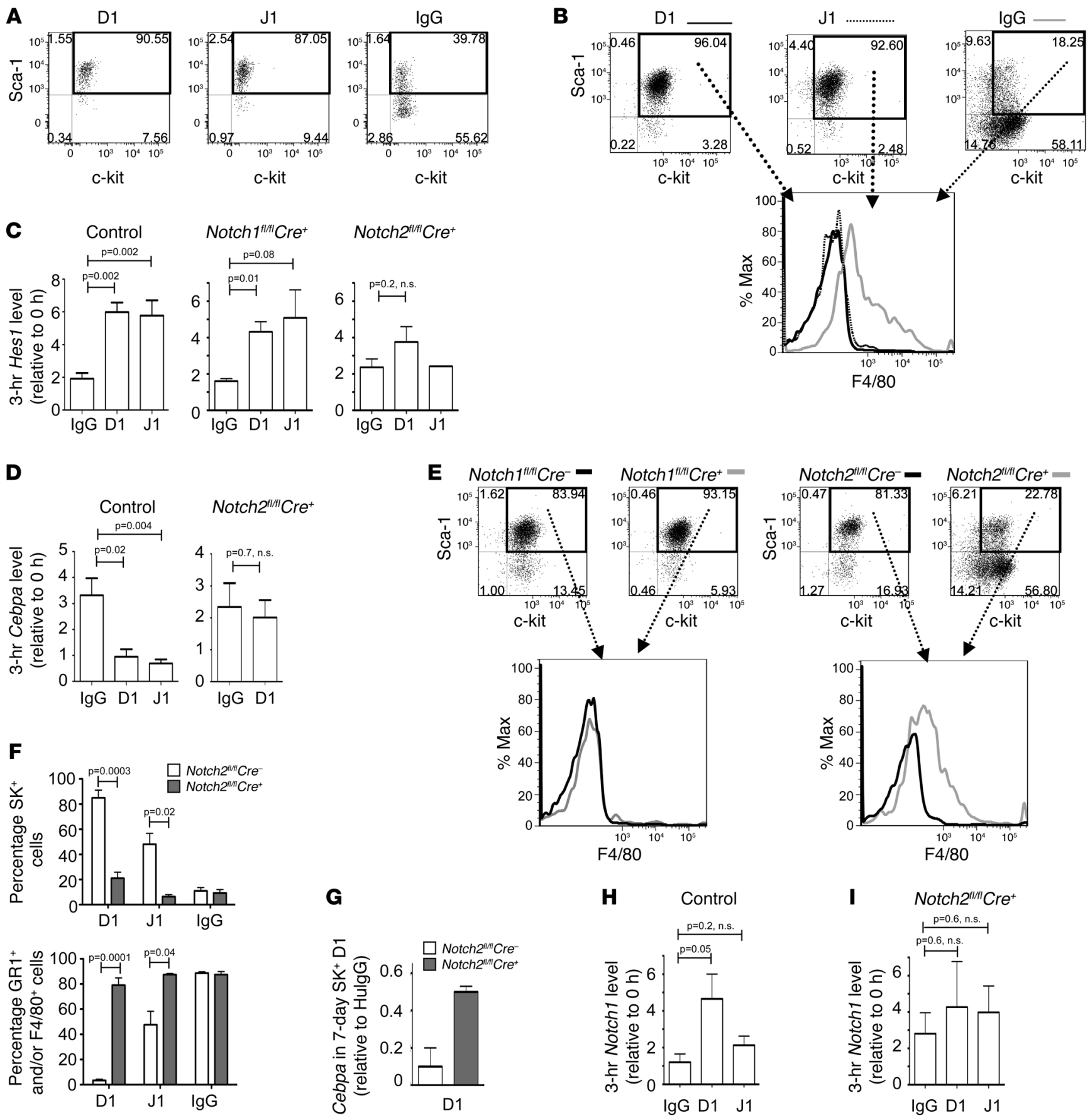

Figure 1

Notch2, but not Notch1, mediates SK+ self-renewal and inhibition of myeloid differentiation with Delta1ext-lgG (D1) and Jagged1ext-lgG (J1). (A and B) Representative dot plots after 3 (A) and 7 (B) days of LSKSP culture with Notch ligand. (C) Hes1 mRNA levels after 3 hours of LSKSP

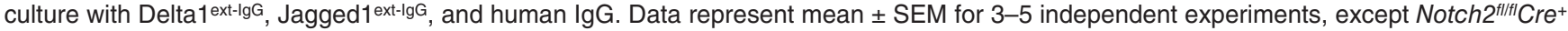
with Jagged1 1 ext-lgg (mean \pm range for 2 independent experiments). $P$ values were derived with 2-tailed Student's $t$ test. (D) Cebpa mRNA levels after 3 hours of LSKSP culture. Data are mean \pm SEM from 5 and 3 independent experiments for control and $\mathrm{Notch}^{t / l f l} \mathrm{Cre}^{+}$, respectively. $P$ values were derived with 2-tailed Student's $t$ test. (E) Representative dot plot after 7 days of LSKSP culture with Delta1 ext-lgG with gated SK ${ }^{+}$ cell expression of F4/80 (histograms). (F) Percent SK+ or GR1 ${ }^{+} F 4 / 80^{+}$cells after 14 days of LSKSP culture. Data represent mean \pm SEM from 4 independent experiments. $P$ values were derived with 2-tailed paired Student's $t$ test. (G) Cebpa mRNA levels in SK+ sorted cells after 7 days of LSKSP culture. Data represent mean \pm range from 2 independent experiments. (H and I) Notch1 mRNA levels after 3 hours of LSKSP culture with Delta1 ${ }^{\text {ext-IgG }}$. Data represent mean \pm SEM from 3-4 independent experiments. $P$ values were derived with 2-tailed Student's $t$ test. Numbers within dot plots denote percentage of events within the respective quadrants. 
A
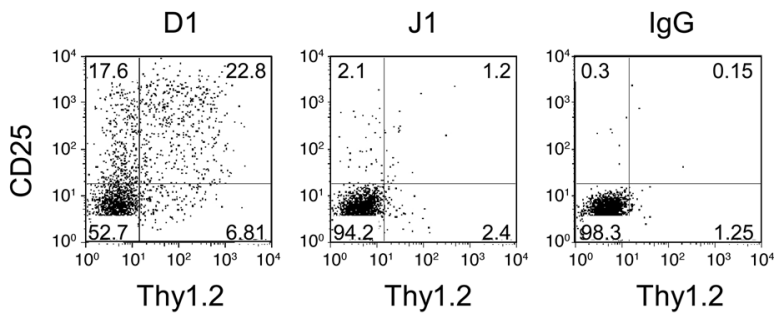

$\mathbf{B}$

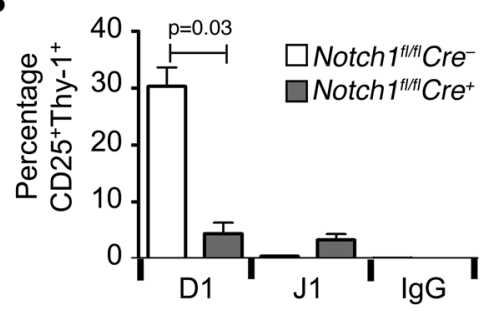

C

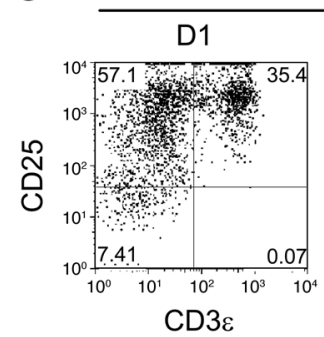

Control shRNA
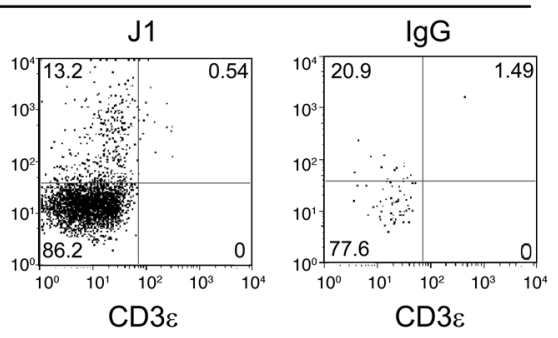

Fringe shRNA
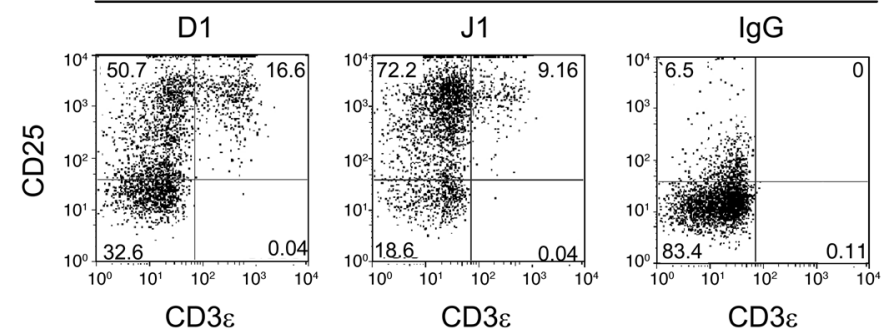

Figure 2

Notch1 mediates generation of CD25+Thy1.2+ progenitors, and Fringe mediates differential Notch1/Notch2 activation. LSKSP cells were cultured with Delta1 ${ }^{\text {ext-lgG }}$, Jagged1 1 ext-IgG, and human IgG. (A) Representative dot plot after 14 days of culture. (B) Percent CD25+Thy $1.2^{+}$progeny generated after 14 days of culture. Data are mean \pm SEM from 3-4 experiments. $P$ values were calculated using 2-tailed paired Student's $t$ test. (C) Representative dot plots from 1 of 2 separate experiments after 18 days of culture of LSKSP cells infected with shRNA. Numbers within dot plots denote percentage of events within the respective quadrants.

Selective activation of Notch2 by Jagged is mediated by Fringe. Hematopoietic differentiation toward the $\mathrm{T}$ cell lineage is known to be dependent upon Notch1 signaling. Previous studies from our group $(19,26)$ and others $(27,28)$ have shown that the differentiation of hematopoietic precursors toward the $\mathrm{T}$ cell lineage is promoted in the presence of Delta family members. Here we showed that culture of control cells with Delta1 ${ }^{\text {ext-IgG }}$ but not Jagged $1{ }^{\text {ext-IgG }}$, induced differentiation toward the $\mathrm{T}$ lineage, as indicated by generation of $\mathrm{CD} 25^{+} \mathrm{Thy} 1.2^{+}$progeny and increased expression of Ptcra mRNA (Figure 2A and data not shown). In addition, Delta-induced differentiation toward the $\mathrm{T}$ lineage was not observed upon culture of Notch1-deficient cells (Figure 2B). Together, these results are consistent with the known requirement for Notch 1 in inducing $\mathrm{T}$ cell differentiation in response to Delta and suggest that subsequent to Notch 1 message upregulation in LSKSP cells, Jagged activates Notch2 but not Notch1. Notch2-deficient LSKSP cells failed to generate $\mathrm{CD} 25^{+} \mathrm{Thy} 1.2^{+}$progeny in the presence of either Notch ligand, perhaps because insufficient Notch signaling leads to rapid myeloid differentiation with loss of competence for $\mathrm{T}$ cell differentiation prior to acquisition of Notch1 signaling.

Previous studies indicated that selective Notch activation in response to Delta versus Jagged is mediated via Fringe (21). To test whether the failure of Jagged $1^{\text {ext-IgG }}$ to induce $T$ cell differentiation was caused by Fringe-mediated inhibition of Notch1 activation, we reduced Fringe levels in LSKSP cells by infection with a GFP lentivirus encoding shRNA designed to inhibit expression of all 3 Fringe homologs: lunatic, manic, and radical (encoded by $L f n g, M f n g$, and $R f n g$, respectively). After 10 days in culture, mRNA levels of $L f n g$ and $M f n g$ were reduced 2- to 3 -fold, and Rfng mRNA was reduced 4-fold, in sorted $\mathrm{GFP}^{+}$cells (Supplemental Figure 1; supplemental material available online with this article; doi:10.1172/JCI43868DS1). Reduced Fringe expression did not affect the percentage of CD25+Thy $1.2^{+}$progeny generated in cultures incubated with Delta $1^{\text {ext-IgG }}$, but did increase the percentage of cells expressing cytoplasmic CD3e

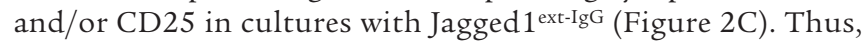
within the LSKSP progeny, the selective effect of Delta $1^{\text {ext-IgG }}$ on $\mathrm{T}$ cell progenitor generation rests in Fringe-mediated inhibition of Notch 1 activation by Jagged $1^{\text {ext-IgG }}$. These findings are consistent with the known requirement for Delta ligands in thymus for induction of $\mathrm{T}$ cell differentiation (25) and suggest a role for the known presence of Jagged in BM for enhancing precursor generation (29).

Neither Notch1 nor Notch2 is required for HSC function in vivo during homeostasis. To assess the role of Notch signaling in stem/progenitor cells within the adult BM, we compared the frequency and number of different BM subsets from mice bearing in vivoinduced Notch1 or Notch2 deletions with those of similarly treated littermates lacking the $M x C r e$ gene. Analysis of BM in homeostasis from control, Notch1-deficient, and Notch2-deficient mice revealed similar numbers of cells as well as normal proportions and numbers of $\mathrm{SK}^{+}$progenitors, cells expressing myeloid markers GR1 and F4/80, and cells expressing the B cell marker B220 (data not shown). Limiting-dilution competitive repopulation transplant analysis using BM in homeostasis indicated similar HSC frequencies from control, Notch1-, and Notch2-deficient mice (Supplemental Tables 1 and 2); furthermore, secondary transplants with mice initially transplanted with Notch2-deficient BM, performed after the primary transplanted BM reached homeostasis (40 weeks after transplant), showed no differences (Supplemental Table 3). These results are consistent with previous studies of homeostatic hematopoiesis showing that ablation of canonical Notch signaling does not affect frequency of functional BM HSC (12). 
A

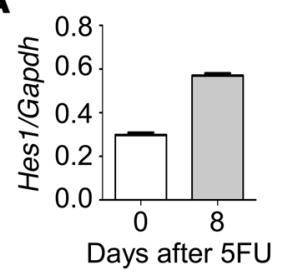

B
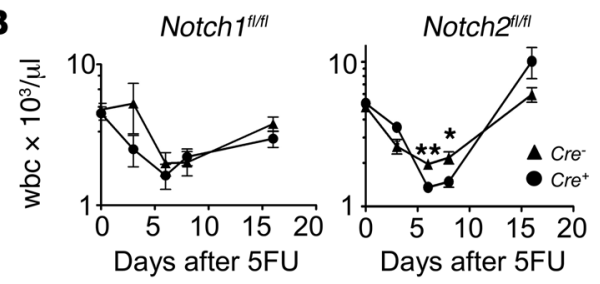

C
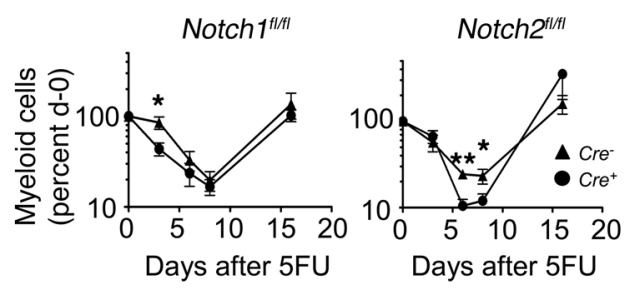

D Notch $1^{\text {itit }}$
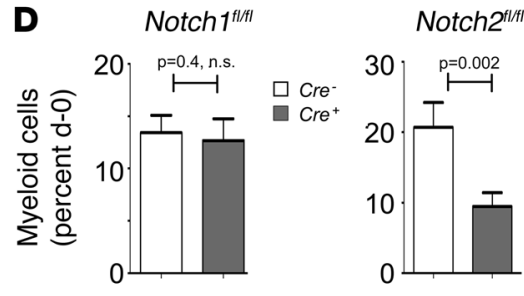

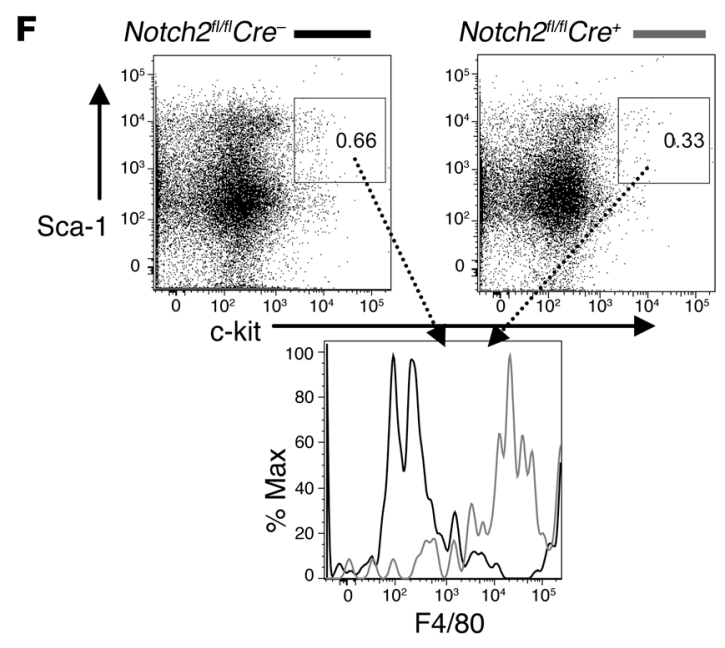

G
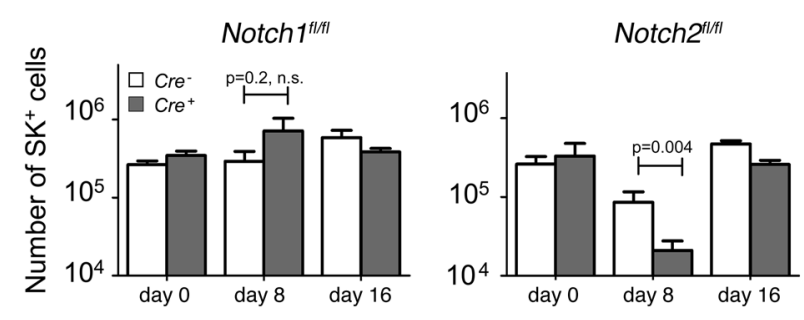

H

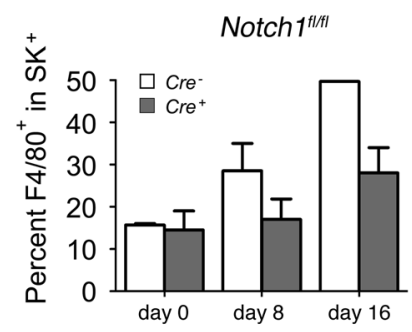

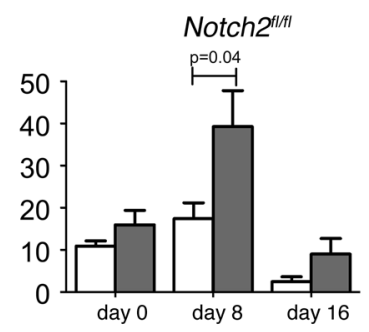

E

Notch $1^{\text {thrf }}$
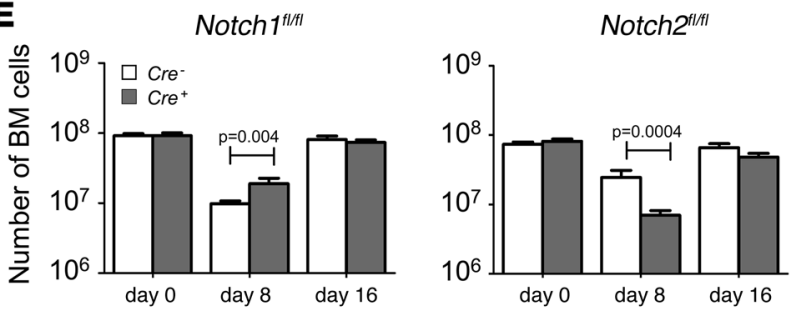

Figure 3

Notch2 modulates BM stress recovery from 5FU. (A) Hes1 mRNA levels in SK+ sorted BM cells 8 days after $5 \mathrm{FU}$ treatment. Data are mean \pm SD of 3 replicates. (B-D) Recovery of PB wbc number and myeloid cell numbers (GR1+ and/or F4/80+; shown relative to day-0 levels) in mice following 5FU treatment. (B and C) Data are mean \pm SEM from 6 (days 3 and 16) or 12 (days 6 and 8) mice from a representative experiment $\left({ }^{\star} P<0.05,{ }^{* *} P<0.01\right)$. (D) Data are mean \pm SEM percent day-0 levels 8 days after $5 \mathrm{FU}$ treatment from 3 independent Notch 1 experiments with 30-32 mice per group and 2 independent Notch2 experiments with 17 mice per group. (E) BM cell number at 0, 8, and 16 days after 5FU. (F) Representative dot plots generated from BM 8 days after $5 F U$. Gated $\mathrm{SK}^{+}$cell expression of F4/80 is shown in the histogram. Numbers represent the proportion of cells within the respective gate. ( $\mathbf{G}$ and $\mathbf{H})$. Number and percentage of $\mathrm{SK}^{+}$gated cells expressing $\mathrm{F} 4 / 80$ at 0,8 , and 16 days after 5FU. (E, G, and $\mathbf{H})$ Notch1 data represent mean \pm SEM from 3 independent experiments with 3 or 6 mice per group (total 17 Notch $1^{f / / f l} C r{ }^{+}$ and 17 Notch1 ${ }^{\text {flffl }} \mathrm{Cre}^{-}$individuals). Notch2 data represent mean \pm SEM from 2 independent experiments with 5 or 6 mice per group (total 11

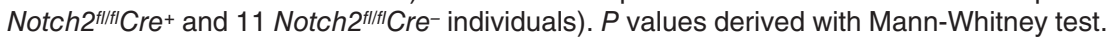

Notch2 modulates hematopoietic recovery following $5 F U$ challenge by retarding myeloid differentiation and enhancing generation of early precursor cells. BM recovery after stress induced by the chemotherapeutic agent 5-fluorouracil (5FU) requires rapid generation of MPPs to efficiently replenish depleted cycling progenitors and cells differentiating along various hematopoietic lineages. A role for Notch signaling in recovery after $5 \mathrm{FU}$ treatment was suggested by the 2-fold increase in Hes1 mRNA in sorted LSK BM cells from mice 
A

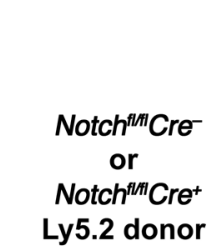

Competitive Secondary BMT:

with $1 \times 10^{5}$

Ly5. 1 competitors

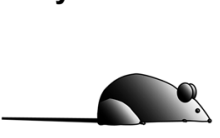

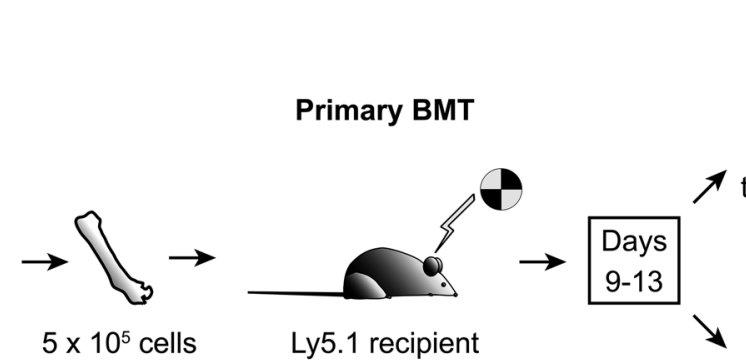

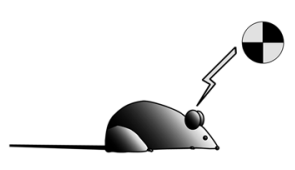

Ly5.1 recipient

Noncompetitive Secondary BMT: without competitors (radioprotection)

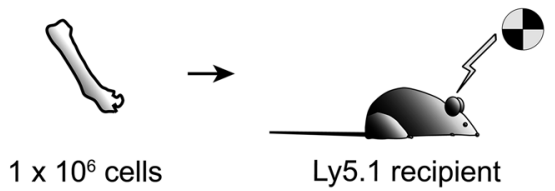

D

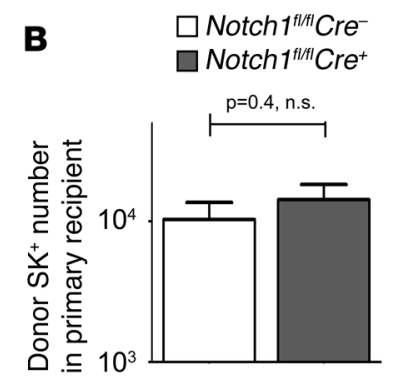
$\square$ Notch2 ${ }^{1 / 4} \mathrm{Cre} \mathrm{Cr}^{-}$
$\square$ Notch $2^{\text {th/flif }} \mathrm{Cre}^{+}$
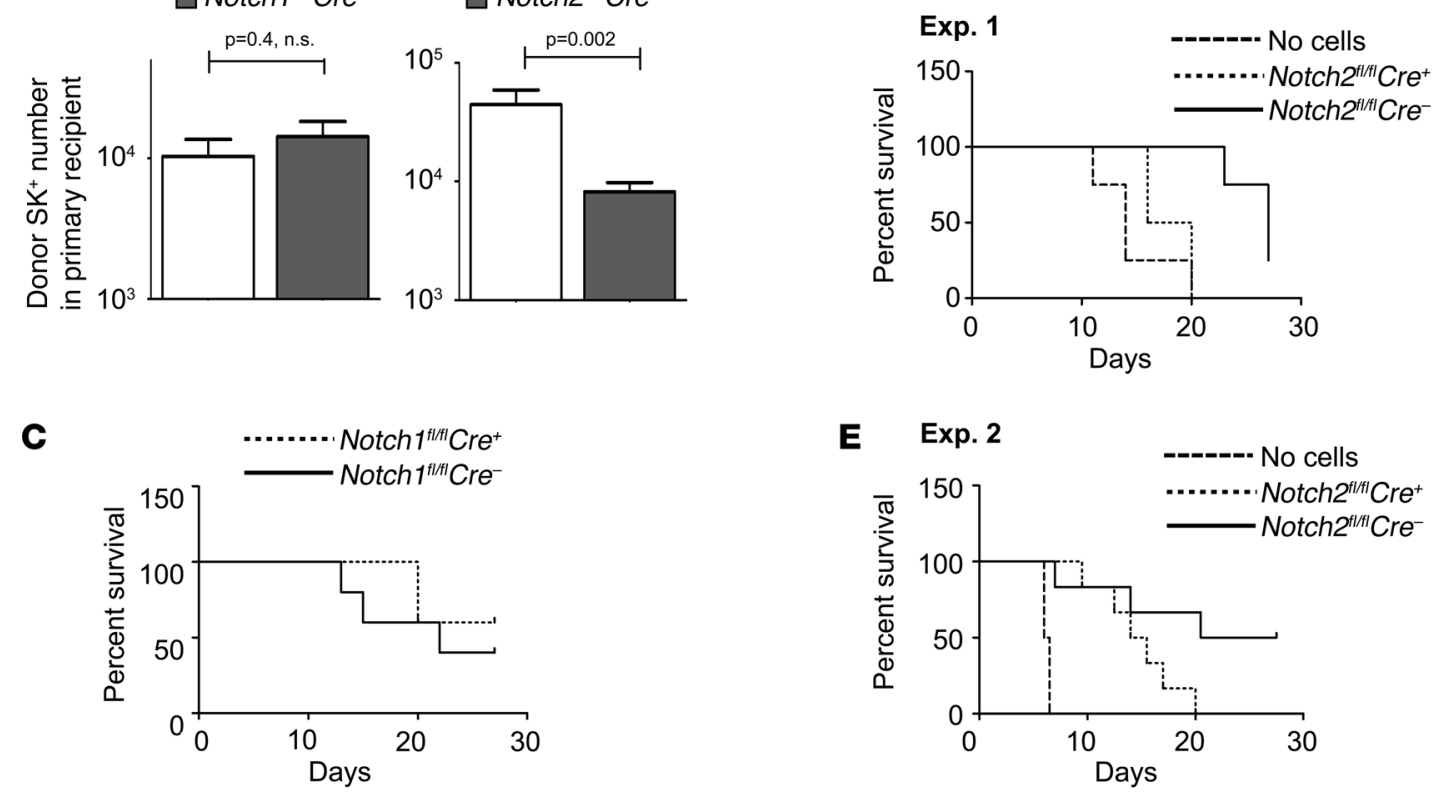

Figure 4

Notch2 enhances the tempo of HSC and MPP recovery after transplant into irradiated recipients. (A) Measurement of HSC and MPP recovery after transplant into irradiated recipients. BMT, BM transplantation. (B) Number of donor (Ly5.2+) and SK+ gated cells generated 13 days after transplant into primary recipients. Data are mean \pm SEM from 2 independent experiments with 5 mice per group per experiment (total 10 Notch2 ${ }^{f / f l} \mathrm{Cre}{ }^{+}$and 10 Notch2 $^{f / f I} \mathrm{Cre}^{-}$). (C) Survival outcome after transplant into lethally irradiated recipients of $1 \times 10^{6}$ cells from primary recipients transplanted 11 days previously with $5 \times 10^{5} \mathrm{Notch} 1^{\mathrm{fl/fI}} \mathrm{Cre} \mathrm{e}^{-}$or $\mathrm{Notch} 1^{\mathrm{fl/fI}} \mathrm{Cre}{ }^{+}$cells. Results were analyzed with a log-rank nonparametric test and expressed as Kaplan-Meier survival curves $(n=4, P=0.45)$. (D) Survival outcome after transplant into lethally irradiated recipients of $1 \times 10^{6}$ cells from primary recipients transplanted 11 days previously with $5 \times 10^{5} \mathrm{Notch}^{\mathrm{fl/fl}} \mathrm{Cre} \mathrm{e}^{-}$or $\mathrm{Notch} 2^{\text {fl/fl}} \mathrm{Cre}{ }^{+}$cells or with no cells. Results were analyzed with a log-rank nonparametric test and expressed as Kaplan-Meier survival curves $(n=4, P=0.01)$. (E) Survival outcome after transplant into lethally irradiated

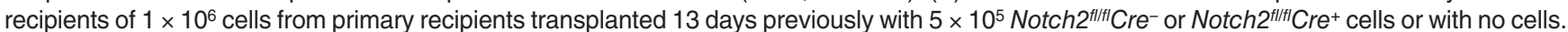
Results were analyzed with a log-rank nonparametric test and expressed as Kaplan-Meier Survival curves $(n=6, P=0.05)$.

that received a single dose of $5 \mathrm{FU} 8$ days previously (Figure 3A). This corroborates previous studies showing reduced Hes 1 in BM LSK progenitors following loss of Notch signaling (11).

To assess the role of increased Notch signaling in recovery from $5 \mathrm{FU}$ stress and to distinguish the involved receptor, we first com- pared recovery in PB from mice with Notch1 or Notch2 deletion. At 7-9 days after 5FU, wbc counts were 2-fold less in Notch2-deficient mice, but were similar or tending toward an increase in Notch1-deficient mice, compared with control littermates (Figure $3 \mathrm{~B})$. Moreover, the percent of myeloid (GR1 $1^{+}$and/or $\mathrm{F} 480^{+}$) 
Table 1

HSC/MPP generation and frequency 2 and 9 weeks after transplant

of lethally irradiated recipients

\begin{tabular}{|c|c|c|c|c|}
\hline & 2 weeks (shor & term MPPs) ${ }^{A}$ & 9 weeks (long & -term HSCs) ${ }^{B}$ \\
\hline & Notch1 $^{t 1 / f I} \mathrm{Cre}^{-}$ & Notch1 $^{t / / f I} \mathrm{Cre}^{+}$ & Notch $1^{f / / f I} \mathrm{Cre}^{-}$ & Notch $^{1 / / 1 / 1} \mathrm{Cre}^{+}$ \\
\hline SC/MPP generation after transplant ( & (positive mice/ & tal mice) & & \\
\hline $5 \times 10^{6} \mathrm{BM}$ cells & $5 / 5$ & $5 / 5$ & $5 / 5$ & $4 / 5$ \\
\hline $1 \times 10^{6} \mathrm{BM}$ cells & $4 / 4$ & $4 / 4$ & $0 / 4$ & $1 / 4$ \\
\hline $2 \times 10^{5} \mathrm{BM}$ cells & $0 / 4$ & $0 / 4$ & $0 / 4$ & $0 / 4$ \\
\hline HSCs/MPPs per $1 \times 10^{6}$ BM cells (no.) & 1.8 & 1.8 & 0.4 & 0.3 \\
\hline Donor cells per primary recipient (no.) & $3.9 \times 10^{6}$ & $5.2 \times 10^{6}$ & $3.9 \times 10^{6}$ & $5.2 \times 10^{6}$ \\
\hline HSCs/MPPs per primary recipient (no. & .) 7.0 & 9.3 & 1.4 & 1.5 \\
\hline
\end{tabular}

Lethally irradiated secondary recipients were transplanted with decreasing numbers of BM cells from

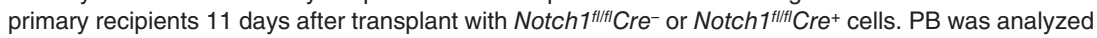
2 and 9 weeks after secondary transplant for the percentage of donor-derived cells. Animals were considered positive for HSCs/MPPs if engraftment in PB was more than $2.0 \%$ donor-derived cells with both lymphoid and myeloid contribution. Poisson statistics were used to calculate HSC/MPP frequency.

${ }^{A} P=0.6$ between groups at the 2 -week time point. ${ }^{B} P=0.7$ between groups at the 9 -week time point. range, \pm 187$)$. These data indicate during the initial 8 days of BM recovery after treatment with $5 \mathrm{FU}$, Notch 2 inhibits myeloid differentiation and promotes generation of short-term repopulating MPPs. However, although we did not detect differences in longterm repopulating HSCs in Notch2-deficient compared with control mice, we suspect that a single dose of 5FU failed to deplete HSCs; to a great extent, they remained quiescent. Hence, we chose to quantify differences in HSC/ MPP recovery after transplantation of control and Notch2-deficient BM into lethally irradiated mice. We harvested BM from transplanted mice at early time points prior to reaching homeostasis, assayed MPP generation using secondary radioprotection assays, and quantified MPP/HSC numbers using

cells in PB after 5FU injection relative to myeloid cell number before injection was reproducibly and significantly lower in injected Notch2-deficient mice, but not significantly different in Notch1-deficient PB, compared with controls (Figure 3, C and D). Platelet counts and rbc counts did not differ between Notch1- or Notch2-deficient mice compared with control littermates during recovery from $5 \mathrm{FU}$ (data not shown). Because differences were not seen with Notch1-deficient mice, we are convinced effects seen with Notch2-deficient mice were due to the loss of Notch2 and not due to Cre expression.

Analysis of BM regeneration in injected mice 8 days after $5 \mathrm{FU}$ revealed 4-fold fewer cells in Notch2-deficient BM, but similar numbers of cells in Notch1-deficient and control BM (Figure 3E). Notch2-deficient cells also consistently had lower proportions of $\mathrm{SK}^{+}$progenitors, whereas Notch1-deficient BM contained proportions similar to those of control BM (Figure $3 \mathrm{~F}$ and data not shown). We also found 4 -fold fewer $\mathrm{SK}^{+}$progenitors in Notch2-deficient BM, whereas $\mathrm{SK}^{+}$progenitor numbers in Notch1-deficient and control BM were similar (Figure 3G). A higher proportion of $\mathrm{SK}^{+}$Notch2-deficient BM cells expressed the myeloid antigen F4/80 than did normal or Notch1-deficient $\mathrm{SK}^{+}$cells (Figure $3 \mathrm{H}$ ), suggestive of rapid differentiation to the myeloid lineage in the absence of Notch2. This evidence of rapid myeloid differentiation of Notch2-deficient precursor cells in vivo is analogous to the above results from ex vivo culture of Notch2-deficient cells with Delta1 ${ }^{\text {ext-IgG }}$.

By 16 days after 5FU treatment, absolute numbers of BM and $\mathrm{SK}^{+}$cells were similar to those seen before 5FU treatment in Notch1- and Notch2-deficient as well as wild-type mice (Figure 3, E and G). Limiting-dilution competitive repopulation transplant experiments were used to measure HSC numbers during recovery from 5FU. Prior to 5FU treatment, the absolute number of HSCs was similar in BM of control mice (903 HSCs; range, \pm 363 ) and Notch2-deficient littermates (655 HSCs; range, \pm 36 ), which suggests that the differences observed during recovery did not originate from differences in the initial number of cells capable of long-term repopulation. Furthermore, at 8 days after 5FU treatment, the absolute number of HSCs did not differ in control (805 HSCs; range, \pm 70 ) and Notch2-deficient mice (524 HSCs; limiting dilution secondary transplantation assays.

Notch2 enhances the tempo of HSC and MPP recovery after transplant of lethally irradiated recipients with a limiting number of BM cells. To further address whether Notch2 is required for HSC or MPP selfrenewal during nonhomeostasis, we monitored BM recovery in lethally irradiated recipients transplanted with control Notch1and Notch2-deficient BM cells at a limiting dose of $5 \times 10^{5} \mathrm{BM}$ cells (Figure 4A). Upon sacrifice after primary transplant, we enumerated stem cell phenotypes as well as HSC numbers in BM of primary recipients. Recovery was evident in mice transplanted with control cells between 11 and 13 days after transplant. Prior to 11 days, BM from mice transplanted with control cells contained a baseline number of $9.1 \pm 0.7 \times 10^{6}$ (SEM) cells, whereas by 13 days, BM from similarly transplanted mice contained significantly increased numbers of cells $\left(13.0 \pm 1.5 \times 10^{6} ; P=0.01\right)$, indicative of recovery. We therefore compared BM from mice 11 days or later and when control BM numbers were higher than the threshold level of $9.1 \times 10^{6}$ cells, to ensure that primary recipients were in recovery. After 13 days, recipients of control cells generated significantly more donor $\mathrm{SK}^{+}$cell numbers than did recipients of Notch2-deficient BM, whereas recipients of control cells and Notch1-deficient cells generated similar $\mathrm{SK}^{+}$cell numbers (Figure 4B). To quantitate long-term repopulating HSCs and short-term repopulating MPPs generated in the recovering $\mathrm{BM}$, competitive repopulating units (CRU) were enumerated in competitive secondary transplants in which BM cells were injected into secondary recipients in limiting dilutions (Figure 4A). Long-term repopulating HSCs contributed to the donor long after 3 weeks, whereas short-term MPPs contributed to PB only in early weeks, and their contribution was minimal after 6 weeks. HSC numbers were similar in control and Notch1-deficient transplanted primary recipients; however, short-term repopulating MPPs (measured after 2 weeks in secondary recipients) and longterm repopulating HSCs (measured after 15 weeks in secondary recipients) were significantly reduced in primary recipients transplanted with Notch2-deficient cells (Tables 1 and 2). Furthermore, lethally irradiated mice receiving $1 \times 10^{6} \mathrm{BM}$ cells from primary recipients previously transplanted with $5 \times 10^{5}$ or $2 \times 10^{6}$ control cells or Notch1-deficient cells survived significantly longer than 


\section{Table 2}

HSC/MPP generation and frequency 2 and 15 weeks after transplant

of lethally irradiated recipients

\begin{tabular}{|c|c|c|c|c|}
\hline & 2 weeks (sho & term MPPs) ${ }^{A}$ & 15 weeks (lor & g-term HSCs) $)^{\mathrm{B}}$ \\
\hline & Notch ${ }^{2 I / / I} \mathrm{Cre}$ & 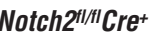 & $\mathrm{Notch}^{\mathrm{fl} / \mathrm{fl}} \mathrm{Cre}^{-}$ & Notch2it/IICre \\
\hline SC/MPP generation after transplant & (positive mice & tal mice) & & \\
\hline $5 \times 10^{6} \mathrm{BM}$ cells & $3 / 3$ & $3 / 3$ & $3 / 3$ & $2 / 3$ \\
\hline $1 \times 10^{6} \mathrm{BM}$ cells & $7 / 7$ & $5 / 7$ & $4 / 7$ & $0 / 7$ \\
\hline $2 \times 10^{5} \mathrm{BM}$ cells & $5 / 6$ & $1 / 7$ & $0 / 6$ & $0 / 7$ \\
\hline $4 \times 10^{4} \mathrm{BM}$ cells & $2 / 7$ & $1 / 7$ & $0 / 7$ & $0 / 7$ \\
\hline HSCs/MPPs per $1 \times 10^{6}$ BM cells (no.) & 8.8 & 1.3 & 0.7 & 0.1 \\
\hline Donor cells per primary recipient (no.) & .) $12.0 \times 10^{6}$ & $7.4 \times 10^{6}$ & $12.0 \times 10^{6}$ & $7.4 \times 10^{6}$ \\
\hline HSCs/MPPs per primary recipient (no. & o.) 105.6 & 9.6 & 8.4 & 0.7 \\
\hline
\end{tabular}

Lethally irradiated secondary recipients were transplanted with decreasing numbers of BM cells from pri-

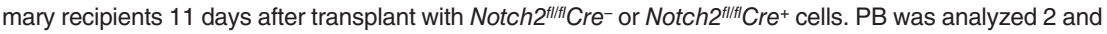
15 weeks after secondary transplant for the percentage of donor-derived cells. Animals were considered positive for HSCs/MPPs if engraftment in PB was more than $2.0 \%$ donor-derived cells with both lymphoid and myeloid contribution. Poisson statistics were used to calculate HSC/MPP frequency. ${ }^{A} P=0.0004$ between groups at the 2 -week time point. ${ }^{B} P=0.02$ between groups at the 15 -week time point.

The importance of limiting signaling via Notch 1 in HSCs is suggested by the loss of HSCs in LRF-deficient mice or in mice transplanted with HSCs that overexpress activated Notch1, presumably as a result of increased $\mathrm{T}$ cell differentiation (31). This effect of the LRF deletion is reversed, however, upon concomitant Notch1 deletion, further suggesting the importance of mechanisms reducing Notch1 signaling in HSCs (32).

Our ex vivo data were consistent with previous studies demonstrating that induction of unique cell fates by Notch ligands is caused by differential activation of Notch receptors by specific ligands $(27,28,33)$. Both Delta $1^{\text {ext-IgG }}$ and Jagged $1^{\text {ext-IgG }}$ induce generation of $\mathrm{SK}^{+}$precursors via activation of Notch2, but, as seen previously with stromal cells express-

did mice receiving $1 \times 10^{6} \mathrm{BM}$ cells from primary recipients previously transplanted with equal numbers of Notch2-deficient cells without competitors. In one experiment, mice receiving Notch2deficient cells survived no longer than irradiated mice receiving no cells (Figure 4, C-E, and Supplemental Figure 2), which indicates that Notch2 is required to generate sufficient numbers of cells, including short-term and possibly long-term repopulating cells, to provide radioprotection.

\section{Discussion}

Our present data revealed a role for Notch2 signaling in the orderly reconstitution of hematopoiesis within the BM space after injury induced by chemotherapy or radiation. Here we show that Notch signaling enhanced the self-renewal of HSCs and MPPs by preventing their depletion caused by rapid differentiation that might result from environmental pressures, such as the presence of high cytokine levels. Hence, Notch signaling assured the rapid establishment of an adequate pool of stem/progenitor cells after BM injury. Although recovery of HSCs and MPPs during nonhomeostasis occurred more rapidly as a result of Notch regulation, no effect of Notch on HSC/MPP numbers during homeostasis occurred, presumably because environmental space constraints in the HSC niche obviate the effects of Notch signaling. These data are consistent with the Notch-dependent increase in HSC numbers observed during coculture of BM-derived LSK cells with endothelial cell layers (18).

Early during HSC culture with Notch ligands, we observed Notch2-mediated molecular changes, such as increased Hes 1 and suppressed Cebpa mRNA expression in association with rapid suppression of a myeloid differentiation program and enhanced MPP generation. Furthermore, Notch 1 was unable to compensate for Notch2 in Notch2-deficient mice. This activation of Notch2, but not Notch1, in HSCs presumably occurs because of reduced expression of Notch 1 and/or factors such as Fringe, which suppress Notch1-mediated signaling in the presence of Jagged $(21,24)$. It is also possible that leukemia/lymphoma-related factor (LRF), a transcriptional repressor recently found to suppress Notch activity, selectively suppresses Notch1-induced signaling (30). ing individual ligands, only Delta $1^{\text {ext-IgG }}$ induces generation of $\mathrm{CD} 25^{+}$Thy $1^{+}$progenitors. We found that these progenitors were generated with Delta $1^{\text {ext-IgG }}$ via Notch1, consistent with in vivo gain- and loss-of-function studies that demonstrate a requirement for Notch 1 in $\mathrm{T}$ cell development as well as with the notion that exposure of BM HSCs and MPPs to Jagged contributes to enhanced progenitor generation, whereas exposure to Delta family members richly expressed in thymus leads to T cell development $(5,6,34)$. Finally, the inability of LSKSP cells lacking Notch2 but expressing Notch1 to generate CD25+Thy $1^{+}$ progenitors ex vivo indicates the requirement for Notch2 signaling in less mature progenitors before the Notch 1 receptor is functional. This may be a result of rapid differentiation toward the myeloid lineage before the increase in Notch1 mRNA levels detected 3 hours after Notch 2 activation with Delta $1^{\text {ext-IgG }}$ or Jagged $1^{\text {ext-IgG }}$. Although HSC progeny in vivo gained Notch1 function in the absence of Notch2 (i.e., Notch2 deletions did not impair T cell development), it is nonetheless possible that the rate of generation of Notch1-expressing cells with $\mathrm{T}$ cell potential is impaired as a result of Notch2 deficiency.

Overall, our results indicate that Notch2 signaling imposes a choice in HSC progeny between differentiation and self-renewal in recovering BM. Augmented self-renewal in the realm of Notch2 signaling is needed to assure generation of increased numbers of lymphomyeloid precursors prior to the formation of differentiated cells that subsequently reconstitute the $\mathrm{BM}$ and $\mathrm{PB}$. Hence, the rapid differentiation toward the myeloid lineage in the absence of Notch 2 generates mature progeny and fewer HSCs and MPPs. Our present findings, together with our previous data suggesting that quantitative differences in Notch signaling account for retardation of myeloid differentiation with generation of MPPs or promotion of differentiation toward the T cell lineage, lead us to hypothesize that Notch2 induces expression of genes that impede myeloid differentiation, whereas Notch 1 produces increased levels of the same genes as well as genes with a higher activation threshold required for initiation of the $\mathrm{T}$ cell program. Whether this is the result of differences in receptor amounts and/or stability or of differences in transcriptional activity 
induced by Notch1- or Notch2-activated intracellular domains remains to be determined. Alternatively, our in vivo results could be explained by a reduced capacity for Notch2-deficient HSCs to efficiently home in secondary transplants. However, we believe this is unlikely, since Notch2-deficient HSCs homed equivalently to wild-type cells - leading to BM recovery - when they were obtained during homeostasis, as indicated by similar frequencies in transplant studies (Supplemental Tables 1 and 3).

Finally, our results point to a requirement for assessing the function of genes in the hematopoietic or other organ systems under nonhomeostatic as well as homeostatic conditions. Genes that are shown to regulate developmental processes but are not required for establishment or maintenance of homeostatic states may also be of practical importance for ex vivo manipulation of stem cell systems, as exemplified by the Notch-mediated expansion of human cord blood hematopoietic progenitor cells capable of rapidly reconstituting BM in patients undergoing HSC transplantation (35).

\section{Methods}

Generation and immobilization of Delta1 $1^{\text {ext-IgG }}$ and Jagged $1^{\text {ext-IgG }}$ and HSC culture. Jagged $1^{\text {ext-IgG }}$ was generated as previously described for preparation of Delta1 ${ }^{\text {ext-IgG }}$ (36). A PCR product was generated using primers encoding appropriate restriction enzyme recognition sequences and the Fc domain of human $I_{g} G_{1}$. Appropriately digested PCR product replaced the myc tags in the previously generated Jagged $1^{\text {ext-myc }}(37)$. Jagged $1^{\text {ext-IgG }}$ protein was produced using NSO cells as previously described for Delta1 ext-IgG (36). Wells of non-tissue culture-treated culture plates (Falcon, BD) were incubated with Delta $1^{\text {ext-IgG }}$ at $5 \mu \mathrm{g} / \mathrm{ml}$, Jagged $1^{\text {ext-IgG }}$ at $20 \mu \mathrm{g} / \mathrm{ml}$, or human $\mathrm{IgG}_{1}$ (Sigma-Aldrich) diluted in PBS together with $5 \mu \mathrm{g} / \mathrm{ml}$ fibronectin fragment CH-296 (Takara Shuzo Co.), incubated overnight at $4{ }^{\circ} \mathrm{C}$, washed extensively, and further incubated with $2 \%$ bovine serum albumin (SigmaAldrich) dissolved in PBS. Equivalence of the concentration of Jagged $1^{\text {ext-IgG }}$ and Delta1 ${ }^{\text {ext-IgG }}$ was determined by ELISA using an HRP-conjugated Fc specific anti-human IgG antibody (Sigma-Aldrich). Cells were cultured as described previously (26).

Cell isolation and immunofluorescence studies. Cell immunophenotype was analyzed by multicolor flow cytometry using an LSR cytometer (BD). Antibodies were purchased from BD Biosciences unless otherwise indicated. Cultured cells were prepared as previously described (37); stained with (a) PE-conjugated monoclonal antibodies against CD25 and F4/80 (eBiosciences), (b) APC-conjugated monoclonal antibodies against c-kit, GR1, and Thy1, and (c) biotinylated antibodies against Sca-1, GR1; and secondarily stained with streptavidin-PerCP. LSKSP cells express levels of Sca-1 and c-Kit and have side population characteristics when stained with Hoechst 33342 (Calbiochem) on BM depleted of the following lineage markers: CD2, CD3, CD8a, CD5, CD11b, B220, GR1, and TER-119. LSKSP cells were obtained using fluorescence-activated cell sorting (FACS) on a Vantage Cell Sorter (BD), and gating was as described previously (38). After each sort, we analyzed sorted populations to confirm that percent $\mathrm{SK}^{+} \mathrm{SP}^{+}$ cells exceeded $90 \%$.

Mice and generation of Notch1- and Notch2-deficient cells. Mice bearing floxed Notch1 and Notch2 genes were maintained and bred with transgenic mice bearing the interferon-inducible Mx1-Cre gene at Fred Hutchinson Cancer Research Center. To induce a deletion in mice bearing Mx1-Cre mutations and Notch $1^{f l / f l}$ and Notch $2^{f l / f l}$ mutations, 2- to 3week-old mice were injected i.p. with $25 \mathrm{mg} / \mathrm{kg}$ solution of poly-I/C (Invivogen). Mice were injected over a 3 -week period in a series of 5 injections every $2-3$ days, followed by a 7 -day break and a second series of 5 injections every 2-3 days. To perform ex vivo deletions, LSKSP cells from Notch $1^{f l / f l}$ or Notch $2^{f l / f l}$ mice were infected with lentivirus encoding Cre recombinase and GFP (Cre-GFP-lenti) at a multiplicity of infection of 500. $\mathrm{GFP}^{+}$cells were isolated after a 3-day culture and replaced. To construct lentivirus encoding Cre and GFP, PCR-amplified Cre with appropriate restriction sites was subcloned into lentivirus. All animal studies were conducted in strict accordance with the NIH guidelines for humane treatment of animals and were approved by the Institutional Animal Care and Use Committee at the Fred Hutchinson Cancer Research Center.

DNA isolation and PCR of genomic DNA. 1 week after the last poly-I/C injection, DNA was extracted from PB of mice using DNA extraction kits according to the manufacturer's instructions (Zymo Research). Genomic Notch1 and Notch2 PCR product amounts were normalized to gene Gja5. Primer sequences for Notch1 (forward, TCATTGTACTGGAACTCTGCACTTT; reverse, TCCACGGTCACCCTTCTCA), Notch2 (forward, CACAGGAGAGGACTGCCAATACT; reverse, TGTCCCGGCTGAGCATGT), and Gjas (forward, ACCATGGAGGTGGCCTTCA; reverse, CATGCAGGGTATCCAGGAAGA) were used to determine copy number of genomic DNA using quantitative Power SYBR-green MasterMix and PCR. To verify that genomic PCR with PBL cells reflected the amount of deletion seen in LSKSP cells, we also measured the amount of deletion in progeny derived from LSK clones sorted from BM cells. LSK clones were placed in individual wells, cultured for 14 days, and genotyped using standard PCR. We found progeny from these clones were $100 \%$ deleted.

RNA isolation and real-time RT-PCR. Total RNA was extracted with TRIzOL. Single-stranded cDNA was synthesized with oligo-dT primer (ThermoScript RT-PCR System; Invitrogen) for 45 minutes at $50^{\circ} \mathrm{C}$. Quantitative PCR was performed using Taqman PCR Master Mix on an ABI PRSIM 7700 sequence detection system (Applied Biosystems). Transcript quantification was performed in triplicate or duplicate for every sample, and expression of each gene was normalized to Gapdh for Taqman PCR. The following Taqman primers (Applied Biosystems) were used: Hes1 (Mm00468601_m1), Cebpa (Mm00514283_s1), Notch1 (Mm00435245_m1), Notch2 (Mm00803077_m1), and housekeeping gene Gapdh (Mm99999915_g1). Sequences of SYBR green primers to measure Fringe expression were as follows: $L f n g$ forward, $5^{\prime}$-CTGCACCATTGGCTACATTG-3'; Lfng reverse, 5'-ATGGGTCAGCTTCCACAGAG-3'; $M$ fng forward, 5'-GCTCCCACTTTGTGGACACT-3'; $M$ fng reverse, 5' -TCCCCTCAAAGACACCGTAG-3'; $R f n g$ forward, 5'-ACACCAATTGCTCTGCTGTG-3'; Rfng reverse, 5'-CTAGGTCGCCCCAGGTAGAT-3'.

Transplant and 5FU injection. Mice were injected i.p. with a single $150-\mathrm{mg} / \mathrm{kg}$ dose of 5FU (source). For transplant experiments, BM cells were collected from Ly5.2 Notch1- and Notch2-deficient mice and injected into lethally irradiated (900-1,000 cGy using a Cesium source) Ly5.1 recipients via the tail vein. FACS analysis of $\mathrm{PB}$ was performed at 3-week intervals after BM transplants. A mouse was considered reconstituted if $2 \%$ or more of $\mathrm{PB}$ was of donor origin and donor myeloid (GR $1^{+}$and/or F4/80 $)$and donor lymphoid (CD19+ and $\mathrm{CD}^{+}$) was detected by 9 weeks after transplant. CRU frequency was estimated using Poisson statistics, and 2-tailed ratio of proportions tests with limit dilution transplants were calculated using L-Calc software (StemCell Technologies).

Statistics. Statistical significance of differences was assessed using 2-tailed paired Student's $t$ test, 2-tailed Student's $t$ test, or Mann-Whitney test as appropriate. A $P$ value of 0.05 or less was considered significant.

\section{Acknowledgments}

The authors thank Cynthia Nourigat, David A. Flowers, Melissa Comstock, LaKeisha Perkins, Stacey Dozono, and Steve Staats for their abundant technical assistance. We also thank Suzanne 
Furuyama, Roland Walter, and Ted Gooley for helpful discussions of the text and/or data. L.M. Halasz was a Howard Hughes Medical Institute Medical Research Training Fellow. This work was supported by NIH grant PO1 HL084205. I.D. Bernstein is an American Cancer Society Clinical Research Professor supported by a gift from the F.M. Kirby Foundation.

Received for publication October 15, 2010, and accepted in revised form December 8, 2010.

1. Wilson A, Laurenti E, Trumpp A. Balancing dormant and self-renewing hematopoietic stem cells. Curr Opin Genet Dev. 2009;19(5):461-468.

2. Li L, Clevers H. Coexistence of quiescent and active adult stem cells in mammals. Science. 2010; 327(5965):542-545.

3. Chiba S. Notch signaling in stem cell systems. Stem Cells. 2006;24(11):2437-2447.

4. Mercher T, et al. Notch signaling specifies megakaryocyte development from hematopoietic stem cells. Cell Stem Cell. 2008;3(3):314-326.

5. Pui JC, et al. Notch 1 expression in early lymphopoiesis influences B versus $\mathrm{T}$ lineage determination. Immunity. 1999;11(3):299-308.

6. Radtke F, et al. Deficient T cell fate specification in mice with an induced inactivation of Notch 1. Immunity. 1999;10(5):547-558.

7. Witt CM, Hurez V, Swindle CS, Hamada Y, Klug CA. Activated Notch2 potentiates CD8 lineage maturation and promotes the selective development of B1 B cells. Mol Cell Biol. 2003;23(23):8637-8650.

8. Witt CM, Won WJ, Hurez V, Klug CA. Notch2 haploinsufficiency results in diminished B1 B cells and a severe reduction in marginal zone B cells. J Immunol. 2003;171(6):2783-2788.

9. Saito T, et al. Notch2 is preferentially expressed in mature $\mathrm{B}$ cells and indispensable for marginal zone $\mathrm{B}$ lineage development. Immunity. 2003;18(5):675-685.

10. Mancini SJ, Mantei N, Dumortier A, Suter U, MacDonald HR, Radtke F. Jagged1-dependent Notch signaling is dispensable for hematopoietic stem cell self-renewal and differentiation. Blood. 2005;105(6):2340-2342.

11. Duncan AW, et al. Integration of Notch and Wnt signaling in hematopoietic stem cell maintenance. Nat Immunol. 2005;6(3):314-322.

12. Maillard I, et al. Canonical notch signaling is dispensable for the maintenance of adult hematopoietic stem cells. Cell Stem Cell. 2008;2(4):356-366.

13. Burns CE, Traver D, Mayhall E, Shepard JL, Zon LI. Hematopoietic stem cell fate is established by the Notch-Runx pathway. Genes Dev. 2005; 19(19):2331-2342.

14. Kunisato A, et al. HES-1 preserves purified hematopoietic stem cells ex vivo and accumulates side population cells in vivo. Blood. 2003;101(5):1777-1783.

15. Stier S, Cheng T, Dombkowski D, Carlesso N,

Address correspondence to: Irwin D. Bernstein, 1100 Fairview Ave. N., PO Box 19024, Seattle, Washington 98109-1024, USA. Phone: 206.667.4886; Fax: 206.667.6084; E-mail: ibernste@fhcrc.org.

Lia M. Halasz's present address is: Harvard Radiation Oncology Program, Boston, Massachusetts, USA.

Mingyi Sun's present address is: Beijing Novo Nordisk Pharmaceuticals Science and Technology Co., Beijing, China.

Scadden DT. Notch1 activation increases hematopoietic stem cell self-renewal in vivo and favors lymphoid over myeloid lineage outcome. Blood. 2002;99(7):2369-2378

16. Varnum-Finney B, et al. Pluripotent, cytokinedependent, hematopoietic stem cells are immortalized by constitutive Notch 1 signaling. Nat Med. 2000;6(11):1278-1281.

17. Yu X, et al. HES1 inhibits cycling of hematopoietic progenitor cells via DNA binding. Stem Cells. 2006;24(4):876-888.

18. Butler JM, et al. Endothelial cells are essential for the self-renewal and repopulation of Notchdependent hematopoietic stem cells. Cell Stem Cell. 2010;6(3):251-264.

19. Dallas MH, Varnum-Finney B, Delaney C, Kato K, Bernstein ID. Density of the Notch ligand Delta1 determines generation of $\mathrm{B}$ and $\mathrm{T}$ cell precursors from hematopoietic stem cells. J Exp Med. 2005;201(9):1361-1366.

20. Ong CT, et al. Target selectivity of vertebrate notch proteins. Collaboration between discrete domains and CSL-binding site architecture determines activation probability. J Biol Chem. 2006;281(8):5106-5119.

21. Hicks C, Johnston SH, di Sibio G, Collazo A, Vogt TF, Weinmaster G. Fringe differentially modulates Jagged 1 and Delta 1 signalling through Notch 1 and Notch2. Nat Cell Biol. 2000;2(8):515-520.

22. Milan M, Cohen SM. A re-evaluation of the contributions of Apterous and Notch to the dorsoventral lineage restriction boundary in the Drosophila wing. Development. 2003;130(3):553-562.

23. Okajima T, Matsuura A, Matsuda T. Biological functions of glycosyltransferase genes involved in Ofucose glycan synthesis. J Biochem. 2008;144(1):1-6.

24. Yang LT, Nichols JT, Yao C, Manilay JO, Robey EA, Weinmaster G. Fringe glycosyltransferases differentially modulate Notch 1 proteolysis induced by Delta 1 and Jagged1. Mol Biol Cell. 2005;16(2):927-942.

25. Visan I, Tan JB, Yuan JS, Harper JA, Koch U, Guidos CJ. Regulation of T lymphopoiesis by Notch1 and Lunatic fringe-mediated competition for intrathymic niches. Nat Immunol. 2006;7(6):634-643.

26. Varnum-Finney B, Brashem-Stein C, Bernstein ID. Combined effects of Notch signaling and cytokines induce a multiple log increase in precursors with lymphoid and myeloid reconstituting ability. Blood.
2003;101(5):1784-1789.

27. Jaleco AC, et al. Differential effects of Notch ligands Delta-1 and Jagged-1 in human lymphoid differentiation. J Exp Med. 2001;194(7):991-1002.

28. Lehar SM, Dooley J, Farr AG, Bevan MJ. Notch ligands Delta 1 and Jagged 1 transmit distinct signals to T-cell precursors. Blood. 2005;105(4):1440-1447.

29. Calvi LM, et al. Osteoblastic cells regulate the haematopoietic stem cell niche. Nature. 2003; 425(6960):841-846.

30. Maeda T, et al. Regulation of B versus T lymphoid lineage fate decision by the proto-oncogene LRF. Science. 2007;316(5826):860-866

31. Chiang MY, Shestove O, Childs ME, Romany C, Aster J, Pear WS. Divergent effects of Notch signaling on Leukemia Stem Cells and Hematotopoitic Stem Cells. ASH Annual Meeting Abstracts. December 5-8, 2009. New Orleans, Lousiana, USA.

32. Lee SU, Maeda M, Sakurai N, Radtke F, Maeda, T. LRF is Indispensable for Hematopoietic Stem Cell Function Via Blocking Notch1-Mediated T CellInstructive Signals in the Bone Marrow Niche. ASH Annual Meeting Abstracts. December 5-8, 2009. New Orleans, Lousiana, USA.

33. Neves H, et al. Effects of Delta 1 and Jagged 1 on early human hematopoiesis: correlation with expression of notch signaling-related genes in CD34+ cells. Stem Cells. 2006;24(5):1328-1337.

34. Wilson A, MacDonald HR, Radtke F. Notch 1deficient common lymphoid precursors adopt a B cell fate in the thymus. J Exp Med. 2001; 194(7):1003-1012.

35. Delaney C, Heimfeld S, Brashem-Stein C, Voorhies H, Manger RL, Bernstein ID. Notch-mediated expansion of human cord blood progenitor cells capable of rapid myeloid reconstitution. Nat Med. 2010;16(2):232-236.

36. Varnum-Finney B, et al. Immobilization of Notch ligand, Delta-1, is required for induction of notch signaling. J Cell Sci. 2000;113 pt 23:4313-4318.

37. Varnum-Finney B, et al. The Notch ligand, Jagged-1, influences the development of primitive hematopoietic precursor cells. Blood. 1998; 91(11):4084-4091.

38. Lin KK, Goodell MA. Purification of hematopoietic stem cells using the side population. Methods Enzymol. 2006;420:255-264. 